Call for Correspondence JNCCN is committed to providing a forum to enhance collaboration between academic medicine and the community physician. We welcome comments about the NCCN Clinical Practice Guidelines in Oncology (NCCN Guidelines), articles published in the journal, or any other topic relating to cancer prevention, detection, treatment, supportive care, or survivorship.

Please send correspondence to JNCCN.edmgr.com or to JNCCN@nccn.org.

Letters should be no more than 400 words, with no more than 5 references if included. Please include the full names, degrees, and affiliations of all letter authors and a phone number or e-mail address for contact.

Letters are considered for publication as space allows. NCCN reserves the right not to publish correspondence for any reason it deems appropriate. All letters are subject to editing and/or abridgment.

\title{
Letter to the Editor: Integrating Skeletal Muscle Mass and Radiodensity Improves Outcome Prediction and Correlation in Oncologic Studies
}

Re: Camila Santos Rodrigues, Gabriela Villaça Chaves. Skeletal Muscle Quality Beyond Average Muscle Attenuation: A Proposal of Skeletal Muscle Phenotypes to Predict ShortTerm Survival in Patients With Endometrial Cancer. J Natl Compr Canc Netw 2018;16(2):153-160.

We read with great interest the recent article by Rodrigues and Chaves ${ }^{1}$ regarding the association of skeletal muscle (SM) phenotypes with short-term survival in patients with endometrial cancer. Understanding the impact of both muscle mass and radiodensity is critically important, and we appreciate the authors' innovative strategy to incorporate both these variables. Given the growing literature on the importance of muscle content and underlying myosteatosis, CT-based body composition assessments should include an assessment of not only SM mass but also muscle radiodensity, a surrogate measurement of muscle composition.

However, we propose an alternative strategy for integrating these important measurements. Rather than dichotomizing participants as high or low radiodensity, why not integrate these 2 variables into 1 continuous variable? Because both muscle mass and radiodensity are defined independently and both are prognostic of many adverse cancer outcomes, we have proposed a mathematical combination of these measures. ${ }^{2}$ Multiplying SM index and the mean SM radiodensity (SMD) from cross-sectional CT imaging at the L3 vertebrae allows for the creation of a new variable, which we have termed "skeletal muscle gauge" (SMG), which retains equal influence of both variables. This method allows for exploring these 2 important muscle components as a single continuous variable with equal weighting rather than creating arbitrary cut points for each. SMG is more highly correlated with aging than either muscle mass or radiodensity alone. ${ }^{2}$

In other recent studies, SMG was shown to be the best predictor of grade $3 / 4$ chemotherapy toxicity in women with early-stage breast cancer undergoing anthracycline and taxane-based chemotherapy. ${ }^{3}$ Other investigators using a similar integrated method for the psoas muscle alone found total psoas gauge was a better predictor of surgical outcomes in patients undergoing radical gastrectomy for gastric cancer. ${ }^{4}$ Although the use of subranges for radiodensity measurements provide ease of presentation and simplifies statistical analysis, maintaining continuous dimensions for radiodensity allow for increased statistical power to detect a relationship between radiodensity and patient outcomes. ${ }^{5}$

Further research is needed to explore the relationship and interplay of both SM mass and radiodensity with adverse outcomes in oncology. With the goal of improved outcome predictions, diagnoses, and treatments, we suggest that future studies evaluate and integrate SMD in a linear rather than dichotomous manner with SM mass.

Grant R. Williams, MD

University of Alabama at Birmingham

Birmingham, $A L$

Email: gwillia@uab.edu

Marc S. Weinberg, PhD

The University of North Carolina

Chapel Hill, NC

Shlomit S. Shachar, MD

The University of North Carolina,

Chapel Hill, NC, and

Rambam Health Care Campus,

Haifa, Israel

doi: 10.6004/jnccn.2018.7041 


\section{References}

1. Rodrigues CS, Chaves GV. Skeletal muscle quality beyond average muscle attenuation: a proposal of skeletal muscle phenotypes to predict shortterm survival in patients with endometrial cancer. J Natl Compr Canc Netw 2018;16:153-160.

2. Weinberg MS, Shachar SS, Muss HB, et al. Beyond sarcopenia: characterization and integration of skeletal muscle quantity and radiodensity in a curable breast cancer population. Breast 2017;24:278-284.

3. Shachar SS, Deal AM, Weinberg M, et al. Body composition as a predictor of toxicity in patients receiving anthracycline and taxane-based chemotherapy for early-stage breast cancer. Clin Cancer Res 2017;23:3537-3543.

4. Lu J, Zheng ZF, Li P, et al. A novel preoperative skeletal muscle measure as a predictor of postoperative complications, long-term survival and tumor recurrence for patients with gastric cancer after radical gastrectomy. Ann Surg Oncol 2018;25:439-448.

5. Altman DG, Royston P. The cost of dichotomis ing continuous variables. BMJ 2006;332:1080.

\section{Author's Reply to Letter to the Editor: Integrating Skeletal Muscle Mass and Radiodensity Improves Outcome Prediction and Correlation in Oncologic Studies}

\begin{abstract}
Author's Reply to Grant R. Williams, Marc S. Weinberg, and Shlomit S. Shachar's Letter to the Editor re: Camila Santos Rodrigues, Gabriela Villaça Chaves. Skeletal Muscle Quality Beyond Average Muscle Attenuation: A Proposal of Skeletal Muscle Phenotypes to Predict Short-Term Survival in Patients With Endometrial Cancer. J Natl Compr Canc Netw 2018;16(2):153-160.
\end{abstract}

We thank the authors for their interest in our results, ${ }^{1}$ and for the opportunity to discuss this important query regarding the need to standardize a method that integrates skeletal muscle (SM) radiodensity and SM quantity in a single variable.

We totally agree with the observation regarding the problems involving loss of statistical power when 2 dichotomic variables are associated to create different classification groups. We are also aware of the methodology created by Weinberg et $\mathrm{al}^{2}{ }^{2}$ which allows the combination of SM radiodensity and quantity. However, despite this as an interesting alternative, we point out some topics for discussion:

- When Aubrey et $\mathrm{al}^{3}$ published a careful review summarizing CT methodologies and factors that influence muscle radiation attenuation, in which they recom- mend that the range between -29 to $+29 \mathrm{HU}$ be evaluated as myosteatosis, we noted that there were no studies dividing SM radiodensity into high or low radiodensity areas. We then pondered that using average muscle attenuation (AMA) to classify myosteatosis could not reflect the total amount of high and low radiation attenuation area in muscles, because the same mean value could represent different proportions of these SM subranges of radiation attenuation. Even as the same body mass index represents different body composition phenotypes, which in turn determines different outcomes, we hypothesized that different SM phenotypes could also determine better or worse prognosis.

- Historically, as our group first showed in a sample of patients with gynecologic cancer who underwent surgical treatment $(n=250)$, high-radiodensity SM index (HRSMI) was the best SM predictor of major surgical complications, although low-radiodensity SM index (LRSMI) and low SM index (SMI) were also predictors. However, in a combined adjustment with low SMI, only HRSMI remained in 
the model as an independent predictor for this outcome (low SMI lost its predictive power). In this study, we did not test AMA as an SM parameter. ${ }^{4}$

- In another recent study, evaluating 1-year mortality in patients with endometrial cancer $(n=232)$, we showed similar results. All of the SM parameters were significantly associated with lower 1-year survival, including AMA. LRSMI presented a trend for significance in the adjusted model. When the SM parameters were additionally adjusted for low SMI, only HRSMI and LRSMI remained in the model as predictors of early mortality, and AMA lost its predictive power. ${ }^{5}$

- These results led us to hypothesize that there is a minimum amount of HRSMI that exerts a protective effect against poor outcomes in oncology, independent of the total amount of SMI. Considering the apparent superiority of HRSMI versus AMA, the initial purpose of our article ${ }^{1}$ was to integrate the phenotypes high or low HRSMI with high or low SMI. However, prior to publication, in addressing a reviewer's suggestion to create the phenotypes using AMA instead of HRSMI in order to facilitate literature comparison, we slightly changed our objectives. Indeed, the results were similar using both strategies.

Although more studies are needed to understand how this interaction between SM quantity and quality occurs, our group has been reflecting on strategies to facilitate the analysis and un- derstanding of these findings. Our main proposal will probably be to calculate the ratio between the subranges of SM radiation attenuation areas (HRSMI and LRSMI) and the total area of the SM (SMI). Comparison of different strategies, including that proposed by Weinberg et $\mathrm{al}^{2}{ }^{2}$ is also anticipated in studies currently underway with our group, for different outcomes. We believe that by using this methodology (HRSMI:SMI ratio), we could integrate SM quantity and quality, maintaining the evaluation of SM quality in terms of area and not the average of attenuation. As both variables have the same unity $\left(\mathrm{cm}^{2} / \mathrm{m}^{2}\right)$, we anticipate that interpretation of the results will be uncomplicated.

Gabriela Villaça Chaves, PhD Brazilian National Cancer Institute, Rio de Janeiro, Brazil Email: gabrielavc@gmail.com doi: 10.6004/jnccn.2018.7042

\section{References}

1. Rodrigues CS, Chaves GV. Skeletal muscle quality beyond average muscle attenuation: a proposal of skeletal muscle phenotypes to predict short-term survival in patients with endometrial cancer. J Nat Compr Canc Netw 2018;16:153-160.

2. Weinberg MS, Shachar SS, Muss HB, et al. Beyond sarcopenia: characterization and integration of skeletal muscle quantity and radiodensity in a curable breast cancer population. Breast J 2017;24:278-284

3. Aubrey J, Esfandiari N, Baracos VE, et al. Measurement of skeletal muscle radiation attenuation and basis of its biological variation. Acta Physiol (Oxf) 2014;210:489-497.

4. de Paula NS, de Aguiar KB, Aredes MA, Chaves GV. Sarcopenia and skeletal muscle quality as predictors of postoperative complication and early mortality in gynecologic cancer. Int J Gynecol Cancer 2018;28:412-420.

5. de Paula NS, Rodrigues CS, Chaves GV. Comparison of the prognostic value of different skeletal muscle radiodensity parameters in endometrial cancer [published online April 25, 2018]. Eur J Clin Nutr, doi: 10.1038/s41430-0180163-5 